\title{
Enhanced bone metabolism in vegetarians - the role of vitamin B12 deficiency
}

\author{
Wolfgang Herrmann ${ }^{1, *}$, Rima Obeid ${ }^{1}$, Heike \\ Schorr' ${ }^{1}$, Ulrich Hübner ${ }^{1}$, Jürgen Geisel ${ }^{1}$, Marga \\ Sand-Hill ${ }^{1}$, Nayyar Ali $^{2}$ and Markus Herrmann ${ }^{1,3}$ \\ ${ }^{1}$ Department of Clinical Chemistry and Laboratory \\ Medicine/Central Laboratory, University Hospital, \\ Saarland University, Homburg/Saar, Germany \\ 2 Diagnostic Laboratory Services, Ministry of Health, \\ Sultanate Oman \\ ${ }^{3}$ ANZAC Research Institute, The University of \\ Sydney, Concord Campus, Concord, Australia
}

\begin{abstract}
Background: Vitamin B12 deficiency and bone fractures are common in vegetarians. However, a direct relationship between vitamin B12 status and bone metabolism in vegetarians has not been tested sufficiently.
\end{abstract}

Methods: Our study included 96 vegetarians (23 German vegans, and 54 German and 19 Indian lacto-, lacto-ovo-vegetarians) and 89 omnivores (Germans and Asian-Indian immigrants in Oman). Blood concentrations of total vitamin B12, holotranscobalamin (holoTC), 25OH-vitamin D (25(OH)D), total homocysteine (tHcy), methylmalonic acid (MMA), and the bone turnover markers (BTMs) bone alkaline phosphatase (BAP), osteocalcin (OC), pro-collagen type I N-terminal peptide (PINP) and C-terminal telopeptides of collagen I (CTx) were measured.

Results: Vegetarians from both population groups exhibited significantly higher concentrations of tHcy, MMA, folate, and BAP, but lower concentrations of holoTC and cobalamin compared with omnivores from the same population. Additionally, German vegetarians had higher circulating activities of BAP as well as higher CTx, OC, and PINP compared with their omnivorous controls. HoloTC and MMA were correlated with OC, CTx and BAP. Subjects with low vitamin B12 status (holoTC $\leq 35 \mathrm{pmol} / \mathrm{L}$ and MMA $>271 \mathrm{nmol} / \mathrm{L}$ ) had significantly lower serum concentrations of $25(\mathrm{OH}) \mathrm{D}$, but higher tHcy and the BTMs $\mathrm{P} 1 \mathrm{NP}, \mathrm{BAP}, \mathrm{OC}$, and CTx, compared with subjects with normal vitamin B12 status. Multiple regression analysis showed that the association between BTMs and markers of vitamin B12 status was independent from the association with $25(\mathrm{OH}) \mathrm{D}$. Approximately $12 \%-14 \%$ of the variation in the concentration of

*Corresponding author: Prof. Dr. Wolfgang Herrmann, University Hospital, Department of Clinical Chemistry and Laboratory Medicine, Saarland University, Building 57, 66424 Homburg/Saar, Germany

Phone: +496841-1630700, Fax: + 496841-1630703,

E-mail: kchwher@uks.eu

Received December 16, 2008; accepted July 22, 2009; previously published online October 12, 2009
BTMs was explained by a regression model including holoTC, MMA and 25(OH)D. The strictness of the diet was not related to the magnitude of change in BTMs. Conclusions: Low vitamin B12 status is related to increased bone turnover in vegetarians which is independent from vitamin D status.

Clin Chem Lab Med 2009;47:1381-7.

Keywords: bone; homocysteine; osteocalcin; vegetarians; vitamin B12; vitamin D.

\section{Introduction}

Osteoporosis is a disease found frequently in elderly persons, often with devastating consequences (1). Recent epidemiologic studies have linked low circulating vitamin B12 and high total homocysteine (tHcy) concentrations to low bone mineral density (BMD) and fractures, typical symptoms of osteoporosis (2-6). Hcy is the final degradation product of the methionine pathway and is increased if vitamin B12, folate and/or B6, important co-enzymes of the Hcy degrading re-methylation and trans-sulfuration pathway, are deficient (7). In vivo and in vitro studies suggest that there are direct effects of Hcy and vitamin B12 on bone metabolism (8-10). In rats, 3 and 5 months of continuous hyperhomocysteinemia causes bone loss and a reduction in bone strength (9). Cell culture experiments using human osteoclasts and osteoblasts revealed a distinct increase in osteoclast activity in the presence of high tHcy and low vitamin B12, B6, and folate concentrations in the culture medium. This was indicated by significant increases in osteoclastic resorption, tartrate resistant acid phosphatase (TRAP) and cathepsin $\mathrm{K}$ activities $(10,11)$. Osteoblast activity was not affected by changes in vitamin B12, B6, and folate concentrations in the culture medium (11). The importance of vitamin B12 for bone health is supported by clinical data where patients with low vitamin B12 status or pernicious anemia exhibited low BMD, increased fracture rates and accelerated bone turnover that could be improved by supplementations with vitamin B12 (12-14). Carmel et al. reported lower levels of bone formation markers including bone alkaline phosphatase (BAP) and osteocalcin (OC) in cobalamin deficient patients, compared to subjects with adequate vitamin status (12).

Vegans are vegetarians who avoid all products of animal origin, such as meat, diary products, and eggs; main sources of vitamin B12 (15). Lacto-, and lactoovo-vegetarians ingest eggs and/or dairy products, which represent a minor source of vitamin B12. Vegans consuming a vitamin B12 free diet over several 
years develop vitamin B12 deficiency $(16,17)$. This deficiency can be associated with a wide spectrum of hematological and neurological symptoms, such as megaloblastic anemia, neuropathies, or cognitive dysfunction $(15,18,19)$. Considering the potential association between vitamin B12 and bone health, it can be suggested that vegetarians may be particularly prone to disturbances in bone metabolism (20). However, studies have reported conflicting data regarding BMD in vegetarians (20-24). The same holds true for bone turnover markers (BTMs). In one study, individuals consuming a raw food vegetarian diet had lower BMD at the hip and lumbar spine, compared to controls ingesting an omnivorous diet (21). However, bone turnover as measured by C-terminal telopeptides of collagen I (CTX) and by BAP did not differ. Contrary, in vegetarian children, reduced serum concentrations of bone formation markers (BAP, OC) by $10 \%-20 \%$, and of bone resorption marker (CTx) by $15 \%$ has been reported (25). Since all these studies did not analyze vitamin B12 status, other factors, such as vitamin $D$ status may have affected the results.

The present study analyzed the association of vitamin B12 status and bone metabolism in vegetarians using biochemical markers of bone turnover and vitamin B12 status.

\section{Materials and methods}

\section{Subjects and study design}

The study population included 122 Germans (35 omnivores and 77 vegetarians). Of the German vegetarians, 23 were vegans and 54 were lacto-, or lacto-ovo-vegetarians. The study also included 73 Asian-Indian immigrants (54 omnivores, 19 lacto-, or lacto-ovo-vegetarians) who were recruited from three different centers from the Sultanate Oman. Asian-Indians were male immigrants who were working in different companies in Oman. People were invited through several central health organizations in Oman to participate. Because the majority of Asian-Indians working in Oman are males, we included only male German subjects in the study. German vegetarians were either vegans or lacto-, and lactoovo-vegetarians who maintained stable dietary habits for at least 2 years. Asian-Indians followed their lacto-, or lacto-ovo vegetarian lifystyles from birth onward. The omnivores were apparently healthy subjects without any history of coronary disease, thrombosis, stroke, or diabetes. Individuals with other chronic or acute diseases that can interfere with Hcy metabolism were not eligible for the study. This included those with depression, dementia, infection, or thyroid dysfunction. Also, people taking B-vitamin supplements, calcium, or vitamin D were not eligible for the study. Written informed consent was obtained from all subjects and the study was approved by the Local Ethical Committees.

Fasting venous blood samples were collected into EDTAcontaining tubes and serum tubes (without anticoagulants). EDTA tubes were centrifuged immediately, while serum tubes were left for $20 \mathrm{~min}$ to clot prior to centrifugation. Then, serum and plasma were separated from cellular blood components and stored at $-80^{\circ} \mathrm{C}$ until measurement. The samples from the Sultanate Oman were shipped on dry ice to the core lab at the Department of Clinical Chemistry of the Medical School at the Saarland University (Germany). Total vitamin B12, holotranscobalamin (holoTC; the bioactive form of vitamin B12), the functional markers of vitamin B12 status [tHcy, cystathionine (Cys) and methylmalonic acid (MMA)], $25(\mathrm{OH})$-vitamin $\mathrm{D}$, folate and the BTMs OC, pro-collagen type I N-terminal peptide (PINP), BAP and CTx were measured. The Diasorin vitamin D assay (DiaSorin Inc, Dietzenbach, Germany) used in this study measures $25 \mathrm{OH}$-vitamin D [25(OH)D2 and D3] [both abbreviated as 25(OH)D].

The concentration of total vitamin B12 in serum was quantified with commercial, competitive chemiluminescence immunoassays (Bayer Diagnostics, Fernwald, Germany) on an ACS Centaur automated analyzer (Bayer Diagnostics, Fernwald, Germany). Intra- and interassay CVs were $<4.4 \%$. HoloTC was measured using an enzymatic fluorescencepolarization-immunoassay from Abbott with an Axsym automated analyzer (Abbott, Wiesbaden, Germany). Intra- and interassay CVs of this method were $<5.1 \%$.

Serum levels of the functional markers of vitamin B12 status tHcy, MMA and Cys were measured using gas-chromatography-mass-spectrometry (GCMS) as described elsewhere (26). Serum concentrations of folate were measured with a commercial, competitive chemiluminescence immunoassay (Bayer Diagnostics, Fernwald, Germany) using an ACS Centaur automated analyzer (Bayer Diagnostics, Fernwald, Germany). Intra- and interassay CVs were $<5.5 \%$.

Serum concentrations of the bone formation markers, OC and PINP, and the bone resorption marker CTx, were measured using electro chemiluminescence immunoassays (Roche Diagnostics, Mannheim, Germany) with an Elecsys 2010 automated analyzer (Roche Diagnostics, Mannheim, Germany). BAP, another bone formation marker, was measured using ELISA (Ostase, IDS, Frankfurt, Germany).

$\mathrm{OC}$ is the most abundant non-collagenous bone matrix protein that is secreted by osteoblasts during bone formation. The OC assay that we used detects the stable N-MID (N-Terminal Midfragment) fragment (amino acid 1-43) of OC by use of two monoclonal antibodies. PINP is cleaved from pro-collagen type I by specific proteases during deposition of collagen I into the bone matrix and is therefore considered a marker of bone formation. BAP, an enzyme that has a role in the mineralization of bone, is specifically produced by osteoblasts. The BAP assay uses a monoclonal antibody against BAP. Intra- and interassay CVs were $<6.5 \%$ for OC, $<3.7 \%$ for PINP and $<6.5 \%$ for BAP.

The bone resorption marker CTx detects crosslinked oligopeptides from the $\mathrm{C}$-terminus of the $\alpha 1$-chain of collagen type I (27). These fragments are released during bone resorption and contain a lysine and an aspartyl residue. While the lysine participates in intermolecular cross-linking, the aspartyl is subject to isomerization. The antibody used in this assay binds only when aspartyl is present in its $\beta$ isomerized form. Intra- and interassay $\mathrm{CVs}$ of the assay were $<4.7 \%$, respectively.

25(OH)D was determined utilizing competitive chemiluminescence immunoassays (DiaSorin, Dietzenbach, Germany). In brief, $25(\mathrm{OH}) \mathrm{D}$ was released from its binding protein and bound to an immobilized 25(OH)D antibody on magnetic particles (solid phase). The binding was in competition with labeled $25(\mathrm{OH}) \mathrm{D}$. Intra- and interassay CVs of the assay were $10.8 \%$ and $13.2 \%$, respectively.

\section{Statistical analysis}

The distribution of all continuous variables was not normal. Therefore, data were log-transformed before applying statistical tests that assume normal distribution. Descriptive statistics are provided as median (10th-90th percentiles). Groups were compared using the Mann-Whitney test or by ANOVA. Binary variables were compared by means of the 
$\chi^{2}$-test. Correlation coefficients were calculated using Spearman's correlation analysis. Backward stepwise multiple regression analysis was performed to investigate determinants of BTMs. All calculations were done using the software package SPSS (Version 17.0 for Windows; SPSS). p-Values below 0.05 were considered statistically significant.

\section{Results}

Table 1 shows the concentrations of B-vitamins and BTMs according to diet and ethnic origin. Concentrations of all blood markers were comparable between German vegans and lacto-lacto-ovo-vegetarians, with the exception of MMA and folate (Table 1). In the German collective, both vegetarian types showed significantly lower vitamin B12 status compared to German omnivores. This was similar to results from Asian-Indians.

German vegans and lacto-, lacto-ovo-vegetarians were considered collectively, and compared to German omnivores. Median concentrations of holoTC and vitamin B12 were markedly lower, and that of MMA was higher, in vegetarians compared to omnivores from both ethnic groups. The proportion of vegetarians from both population groups who had holoTC $\leq 35 \mathrm{pmol} / \mathrm{L}$ and MMA $>271 \mathrm{nmol} / \mathrm{L}(66 \%$ of the Germans, and $69 \%$ of the Asian-Indians; $p=0.547$ ) were comparable. In contrast, the median concentration of serum folate was significantly higher in vegetarians compared with omnivores. Higher folate concentrations were found in Asian-Indian vegetarians compared to German vegetarians.

Bone turnover was accelerated significantly in German vegetarians as shown by higher circulating CTx, OC and BAP concentrations, when compared to German omnivores. There were no significant differences in the concentration of BTMs between German vegans and lacto-, lacto-ovo-vegatarians. Comparing $25(\mathrm{OH}) \mathrm{D}$ between vegetarians and non-vegetarians showed some differences between these two populations. In Germans, 25(OH)D concentrations did not differ according to diet (vegan, lacto-, lacto-ovo-vegetarians and omnivores). In Asian-Indians, 25(OH)D was lower in vegetarians compared to omnivores. In addition, regardless of diet, Asian-Indians had higher 25(OH)D compared to Germans, probably due to differences in exposure to sun light.

We stratified our subjects according to vitamin B12 status (using holoTC and MMA) into deficient and non-deficient groups. Subjects deficient in vitamin B12 were those who had holoTC $\leq 35 \mathrm{pmol} / \mathrm{L}$ and MMA $>271 \mathrm{nmol} / \mathrm{L}$ (Table 2). Irrespective of the ethnic origin, vitamin B12 deficient individuals $(n=63 ; 17 \%$ of them Asian-Indians) showed higher serum concentrations of thcy as well as lower $25(\mathrm{OH}) \mathrm{D}$ concentrations. P1NP, BAP, OC, and CTx were significantly higher in vitamin B12 deficient individuals, indicating increased bone turnover.

Backward regression analyses were applied to find determinants of BTMs (P1NP, BAP, OC) in both ethnic groups. In both populations, markers of B-vitamin status (MMA, holoTC, or tHcy) were significant factors

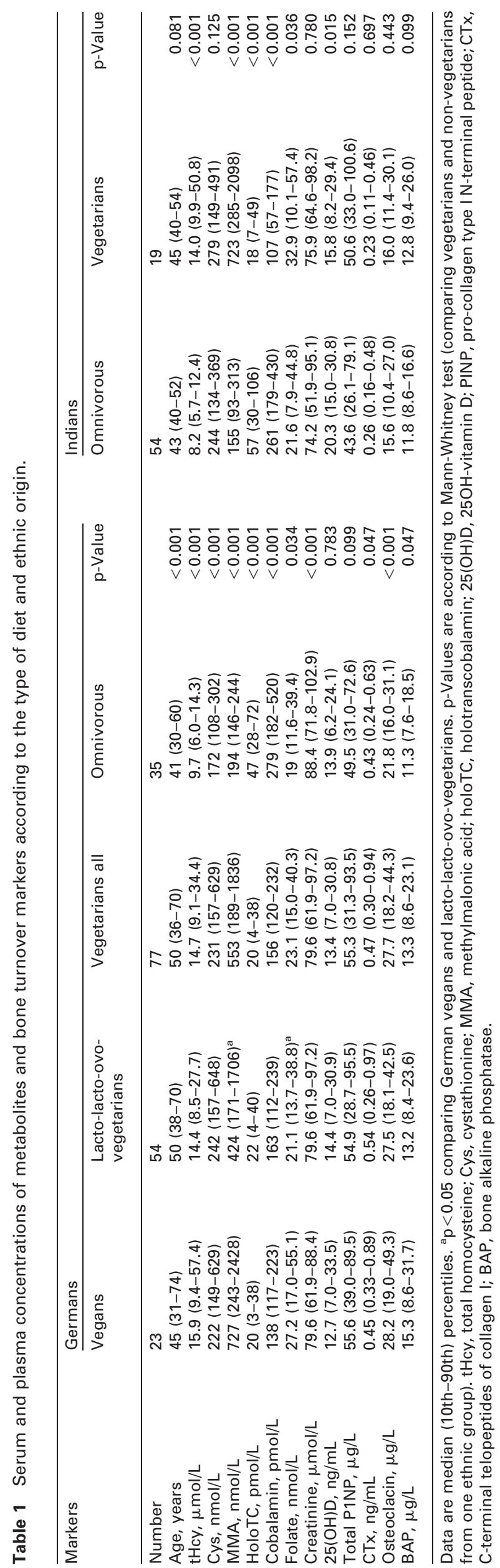


Table 2 Concentrations of metabolites and bone turnover markers according to vitamin B12 status.

\begin{tabular}{|c|c|c|c|c|}
\hline $\begin{array}{l}\text { Metabolites and } \\
\text { bone marker }\end{array}$ & $\begin{array}{l}\text { Normal MMA and } \\
\text { normal holoTC } \\
\mathrm{n}=66\end{array}$ & $\begin{array}{l}\text { Elevated MMA } \\
\text { and low holoTC } \\
n=63\end{array}$ & p-Value (ANOVA) & $p-$ Value $^{a}$ \\
\hline Age, years & $43(35-55)$ & $45(34-70)$ & 0.016 & \\
\hline $\mathrm{tHcy}, \mu \mathrm{mol} / \mathrm{L}$ & $9.5(5.6-13.2)$ & $17.1(10.1-49.8)$ & $<0.001$ & $<0.001$ \\
\hline Cys, nmol/L & 198 (122-355) & 252 (158-609) & 0.001 & 0.015 \\
\hline MMA, nmol/L & 177 (115-242) & 803 (345-2088) & - & - \\
\hline HoloTC, pmol/L & $55(37-102)$ & $16(4-29)$ & - & - \\
\hline Cobalamin, pmol/L & $287(168-514)$ & $141(92-192)$ & $<0.001$ & 0.001 \\
\hline Folate, $\mathrm{nmol} / \mathrm{L}$ & $21.7(10.4-42.4)$ & $22.9(14.0-38.9)$ & 0.551 & 0.194 \\
\hline Creatinine, mg/dL & $0.98(0.76-1.16)$ & $0.90(0.70-1.10)$ & 0.004 & 0.004 \\
\hline 25(OH)D, ng/mL & $18.4(9.3-31.2)$ & $13.0(7.0-30.0)$ & 0.001 & 0.001 \\
\hline Total P1NP, $\mu \mathrm{g} / \mathrm{L}$ & $48(29-77)$ & $55(28-91)$ & 0.057 & 0.031 \\
\hline $\mathrm{BAP}, \mu \mathrm{g} / \mathrm{L}$ & $12.0(8.1-16.7)$ & $13.3(8.6-23.5)$ & 0.012 & 0.008 \\
\hline $\mathrm{OC}, \mu \mathrm{g} / \mathrm{L}$ & $18.6(12.4-29.7)$ & $26.5(14.4-42.2)$ & $<0.001$ & $<0.001$ \\
\hline CTx, ng/mL & $0.35(0.17-0.60)$ & $0.44(0.17-0.86)$ & 0.052 & 0.003 \\
\hline Vegetarians, $\mathrm{n}^{\mathrm{b}}$ & 6 & 62 & $<0.001$ & - \\
\hline
\end{tabular}

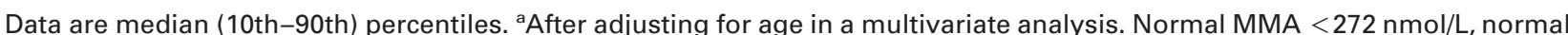
holoTC $>35 \mathrm{pmol} / \mathrm{L}$. ${ }^{\mathrm{b}} \chi^{2}$-test. tHcy, total homocysteine; Cys, cystathionine; MMA, methylmalonic acid; holoTC, holotranscobalamin; 25(OH)D, 25OH-vitamin D; PINP, pro-collagen type I N-terminal peptide; BAP, bone alkaline phosphatase; OC, osteocalcin; CTx, C-terminal telopeptides of collagen I.

explaining the variations in concentrations of BAP and PINP (Table 3). Variations in the concentrations of $O C$ in Asian-Indians were not explained by any of the markers measured in this study.

Concentrations of MMA and BAP correlated positively in both ethnic groups of vegetarians and nonvegetarians (Figure 1). The correlation was significant in Germans $(R=0.23, p=0.011)$ and tended to be significant in Asian-Indians ( $R=0.23, p=0.067$ ) (Figure 1).

\section{Discussion}

The present study assessed the vitamin B12 status of vegetarians and omnivorous subjects using functional biochemical markers, such as holoTC, tHcy and MMA. All of these markers showed one or more significant relationships with markers of bone formation, such as BAP, PINP and OC in the two different ethnic groups. None of the vegetarians included in this study had clinical symptoms of vitamin B12 deficiency. The type of vegetarian diet (vegan or lacto-ovo) had no additional impact on bone turnover, suggesting that mild to moderate vitamin B12 deficiency might be sufficient to increase bone turnover.
Our data support a significant role for vitamin B12 in bone metabolism. In contrast to previous studies $(2,28)$, the majority of vegetarians in our study exhibited insufficient vitamin B12, with the lowest concentrations seen in vegans (Table 1). The EPIC-Oxford study included 9420 vegetarians and 1126 showed an increased risk of fracture (29). Since vegans usually have lower vitamin B12 status compared with lactoovo vegetarians, it can be speculated that vitamin B12 needs to fall below a certain threshold to be clinically relevant. Our study does not confirm this hypothesis since vegans had BTM levels comparable to those seen in lacto-ovo vegetarians. However, it should be noted that the duration, rather than the type of diet, probably determines bone turnover. Since animal products are a good source of vitamin D and vitamin B12, a vegetarian diet might be associated with depletion of both vitamin B12 and vitamin D stores; the latter being of major importance for bone health. Increased bone turnover with aging is associated with accelerated bone loss (30-34).

The first evidence for the relationship between a vegetarian life style, low vitamin B12 status and low BMD was derived from a cross-sectional study of adolescents who were fed a macrobiotic diet up to the

Table 3 Stepwise backward regression analyses showing determinants of concentration of bone turnover markers.

\begin{tabular}{lll}
\hline Dependent variables & $\begin{array}{l}\text { Variable with } \\
\text { significant effect }\end{array}$ & $\beta$-Intercept, $\mathrm{p}$-value \\
\hline In Germans & tHcy & $\beta=0.28, \mathrm{p}=0.001$ \\
PINP & HoloTC & $\beta=-0.10, \mathrm{p}=0.027$ \\
BAP & HoloTC & $\beta=-0.09, \mathrm{p}=0.010$ \\
OC & MMA & $\beta=0.31, \mathrm{p}=0.033$ \\
In Asian Indians & MMA & $\beta=0.10, \mathrm{p}=0.039$ \\
PINP & - & - \\
BAP & & \\
OC & &
\end{tabular}

In each model shown in this Table, independent variables were: holoTC, 25(OH)D, tHcy, and MMA. PINP, pro-collagen type I $\mathrm{N}$-terminal peptide; tHcy, total homocysteine; BAP, bone alkaline phosphatase; holoTC, holotranscobalamin; OC, osteocalcin; MMA, methylmalonic acid. 


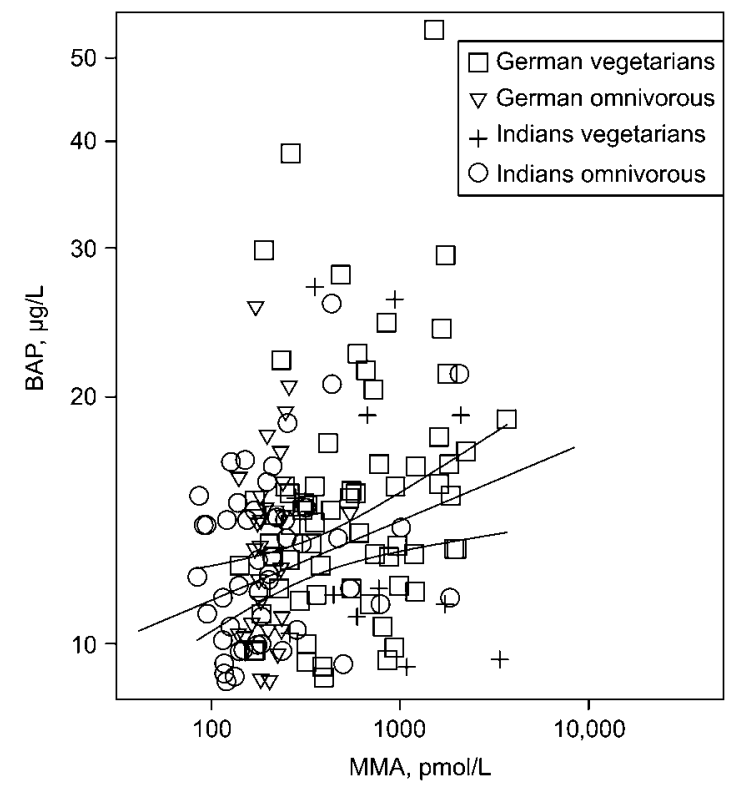

Figure 1 Scatter plot showing the correlation between BAP concentrations and MMA according to diet and ethnicity. Correlation coefficients were, $R=0.23, p=0.011$ in Germans, and $R=0.23, p=0.067$ in Asian-Indians.

age of 6 years, followed by a lacto-(-ovo-) vegetarian or omnivorous diet (22). Total vitamin B12, BMD and bone mineral content (BMC) were lower and MMA was higher in the formerly macrobiotic-fed individuals compared with controls. After adjusting for confounding factors, serum MMA was significantly higher and total vitamin B12 lower in subjects with low BMD compared with subjects with normal BMD. Consistent with this, the Longitudinal Aging Study Amsterdam (LASA) study reported a significant relationship between vitamin B12 and BMD, measured by calcaneal broadband ultrasound attenuation (BUA) in women (2). The strongest evidence for accelerated bone loss in vegetarians comes from the EPIC-Norfolk study which included over 11,000 men and women (35). Male vegetarians exhibited a $6 \%-15 \%$ reduction in BUA, indicating a significant deterioration in bone composition in vegetarians. Previous studies suggested lower body weight as well as lower intake of protein, calcium, vitamin $\mathrm{D}$ and acid substances as potential causative factors for reduced BMD and an increased fracture rate in vegetarians and vegans (20-22, 29, 35). However, in the LASA and the EPIC studies the relationship between BMD/BUA and vegetarian life style, as well as vitamin B12 status, remained significant after adjusting for body weight, food intake and nutrients.

While the association between a vegetarian life style and BMD has been addressed in several studies, there is limited data regarding the association between vitamin B12 status and bone metabolism in vegetarians $(21,22,25)$. The present study provides evidence showing a significant increase in BAP, OC, PINP and CTx in vegetarians. Our results are in contrast to a previous cross-sectional study that compared vegetarians and non-vegetarians. Although $\mathrm{BMD}$ and bone mineral content were lower in vege- tarians, BTMs CTx and BAP, as well as vitamin D concentrations were similar in vegetarians and controls (21). Considering the small number of subjects and the high variability of OC and CTx, the lack of a significant difference is not surprising $(27,36)$. Another recent study that investigated 50 vegetarian children aged 2-10 years found the serum concentrations of BAP, CTx and OC to be $10 \%-20 \%$ lower than the omnivorous controls (25). Since bone metabolism in growing bones of children differs significantly from the situation in adults, comparison of these results with our data is significantly limited $(27,36)$.

Our results are supported by the large epidemiologic LASA study. In this study, women with total vitamin B12 concentrations $<200 \mathrm{pmol} / \mathrm{L}$ and tHcy concentrations $>15 \mu \mathrm{mol} / \mathrm{L}$ had higher concentrations of serum OC and urinary desoxypyridinoline crosslinks (another bone resorption marker representing collagen type I breakdown) compared to women with vitamin B12 levels $>200 \mathrm{pmol} / \mathrm{L}$ and tHcy concentrations $<15 \mu \mathrm{mol} / \mathrm{L}$ (2). In men, no such differences were found.

Our current findings are supported by previous studies. Vitamin B12 has been shown to stimulate proliferation and functional maturation of human bone marrow stromal osteoprogenitor cells (hBMSC) and UMR106 osteoblastic cells, as assessed by [3H]thymidine incorporation and BAP activity (37). Furthermore, in-vitro studies using human osteoclasts revealed activation of osteoclasts with decreasing concentrations of $\mathrm{B} 12, \mathrm{~B} 6$ or folate and increasing concentrations of tHcy. This suggests a causal relationship between decreased B-vitamins and bone degradation (38). However, no such effects have been observed for human osteoblasts (11). While results for osteoblasts are conflicting, existing data support a regulatory role for vitamin B12 in bone resorption.

The mechanisms explaining the relationship between vitamin B12 deficiency and bone turnover is not well understood. A potential link between these two might be the increases in tHcy in vitamin B12 deficient subjects (Table 3). Hcy has repeatedly been shown to adversely affect bone, with stimulation of osteoclasts, inhibition of osteoblasts and disturbances in collagen crosslinking being potential mechanisms (39). In addition, a recent animal study by our group (40) showed bone-specific accumulation of Hcy in hyperhomocysteinemic animals, which supports the potential role of Hcy for bone health.

The differences in vitamin D status between these two populations deserve further investigation. We did not collect data on the dietary intake of the $D$ vitamins and vitamin B12. However, in contrast to the situation in Asian-Indians, 25(OH)D did not differ between German omnivorous and vegetarian subjects. It seems plausible that these differences in Asian-Indians are related to a vegetarian diet, whereas in Germans, decreased exposure to sun is the limiting factor.

In conclusion, the results of the present study show that metabolic signs of vitamin B12 deficiency are related to accelerated bone turnover in vegetarians, 
which may potentially have adverse effects on bone health in these individuals. Concentrations of BTMs were not related to the type of vegetarian diet. Further studies need to investigate whether vitamin B12 supplementation alone or in combination with vitamin $D$ can improve bone turnover and bone health in vegetarians.

\section{Conflict of interest statement}

The authors disclose all sources of financial support for the study. The authors have no conflict of interest.

\section{References}

1. Cummings SR, Melton LJ. Epidemiology and outcomes of osteoporotic fractures. Lancet 2002;359:1761-7.

2. Dhonukshe-Rutten RA, Pluijm SM, de Groot LC, Lips P, Smit JH, van Staveren WA. Homocysteine and vitamin B12 status relate to bone turnover markers, broadband ultrasound attenuation, and fractures in healthy elderly people. J Bone Miner Res 2005;20:921-9.

3. van Meurs JB, Dhonukshe-Rutten RA, Pluijm SM, van der Klift $M$, de Jonge R, Lindemans J, et al. Homocysteine levels and the risk of osteoporotic fracture. N Engl J Med 2004;350:2033-41.

4. Dhonukshe-Rutten RA, Lips M, de JN, Chin APM, Hiddink GJ, Van DM, et al. Vitamin B-12 status is associated with bone mineral content and bone mineral density in frail elderly women but not in men. J Nutr 2003;133:801-7.

5. McLean RR, Jacques PF, Selhub J, Fredman L, Tucker $\mathrm{KL}$, Samelson EJ, et al. Plasma B vitamins, homocysteine, and their relation with bone loss and hip fracture in elderly men and women. J Clin Endocrinol Metab 2008;93:2206-12.

6. McLean RR, Jacques PF, Selhub J, Tucker KL, Samelson EJ, Broe KE, et al. Homocysteine as a predictive factor for hip fracture in older persons. N Engl J Med 2004;350: 2042-9.

7. Finkelstein JD. Pathways and regulation of homocysteine metabolism in mammals. Semin Thromb Hemost 2000:26:219-25.

8. Ozdem S, Samanci S, Tasatargil A, Yildiz A, Sadan G, Donmez $L$, et al. Experimental hyperhomocysteinemia disturbs bone metabolism in rats. Scand J Clin Lab Invest 2007;67:748-56.

9. Herrmann M, Wildemann B, Claes L, Klohs S, Ohnmacht M, Taban-Shomal O, et al. Experimental hyperhomocysteinemia reduces bone quality in rats. Clin Chem 2007; 53:1455-61

10. Herrmann M, Schmidt J, Umanskaya N, Colaianni G, Al MF, Widmann $T$, et al. Stimulation of osteoclast activity by low B-vitamin concentrations. Bone 2007;41:584-91.

11. Herrmann M, Umanskaya N, Wildemann B, Colaianni G, Schmidt J, Widmann T, et al. Accumulation of homocysteine by decreasing concentrations of folate, vitamin B12 and B6 does not influence the activity of human osteoblasts in vitro. Clin Chim Acta 2007;384:129-34.

12. Carmel R, Lau KH, Baylink DJ, Saxena S, Singer FR. Cobalamin and osteoblast-specific proteins. N Engl J Med 1988;319:70-5.

13. Melton ME, Kochman ML. Reversal of severe osteoporosis with vitamin B12 and etidronate therapy in a patient with pernicious anemia. Metabolism 1994;43: $468-9$
14. Goerss JB, Kim CH, Atkinson EJ, Eastell R, O'Fallon WM, Melton LJ III. Risk of fractures in patients with pernicious anemia. J Bone Miner Res 1992;7:573-9.

15. Herrmann W, Geisel J. Vegetarian lifestyle and monitoring of vitamin B-12 status. Clin Chim Acta 2002;326: 47-59.

16. Herrmann W, Schorr H, Obeid R, Geisel J. Vitamin B-12 status, particularly holotranscobalamin II and methylmalonic acid concentrations, and hyperhomocysteinemia in vegetarians. Am J Clin Nutr 2003;78:131-6.

17. Herrmann W, Schorr H, Purschwitz K, Rassoul F, Richter V. Total homocysteine, vitamin B-12, and total antioxidant status in vegetarians. Clin Chem 2001;47:1094-101.

18. Obeid R, Geisel J, Schorr H, Hubner U, Herrmann W. The impact of vegetarianism on some haematological parameters. Eur J Haematol 2002;69:275-9.

19. Obeid R, McCaddon A, Herrmann W. The role of hyperhomocysteinemia and B-vitamin deficiency in neurological and neuropsychiatric diseases. Clin Chem Lab Med 2007;45:1590-606.

20. Chiu JF, Lan SJ, Yang CY, Wang PW, Yao WJ, Su LH, et al. Long-term vegetarian diet and bone mineral density in postmenopausal Taiwanese women. Calcif Tissue Int 1997;60:245-9.

21. Fontana L, Shew JL, Holloszy JO, Villareal DT. Low bone mass in subjects on a long-term raw vegetarian diet. Arch Intern Med 2005;165:684-9.

22. Dhonukshe-Rutten RA, Van DM, Schneede J, de Groot LC, van Staveren WA. Low bone mineral density and bone mineral content are associated with low cobalamin status in adolescents. Eur J Nutr 2005;44:341-7.

23. Ellis FR, Holesh S, Ellis JW. Incidence of osteoporosis in vegetarians and omnivores. Am J Clin Nutr 1972;25: $555-8$.

24. Wang YF, Chiu JS, Chuang MH, Chiu JE, Lin CL. Bone mineral density of vegetarian and non-vegetarian adults in Taiwan. Asia Pac J Clin Nutr 2008;17:101-6.

25. Ambroszkiewicz J, Klemarczyk W, Gajewska J, Chelchowska M, Laskowska-Klita T. Serum concentration of biochemical bone turnover markers in vegetarian children. Adv Med Sci 2007;52:279-82.

26. Herrmann W, Schorr H, Bodis M, Knapp JP, Muller A, Stein G, et al. Role of homocysteine, cystathionine and methylmalonic acid measurement for diagnosis of vitamin deficiency in high-aged subjects. Eur J Clin Invest 2000;30:1083-9.

27. Herrmann M, Seibel M. The amino- and carboxyterminal cross-linked telopeptides of collagen type I, NTX-I and CTX-I: a comparative review. Clin Chim Acta 2008;393: 57-75.

28. Gjesdal CG, Vollset SE, Ueland PM, Refsum H, Meyer HE, Tell GS. Plasma homocysteine, folate, and vitamin B12 and the risk of hip fracture: the hordaland homocysteine study. J Bone Miner Res 2007;22:747-56.

29. Appleby P, Roddam A, Allen N, Key T. Comparative fracture risk in vegetarians and nonvegetarians in EPICOxford. Eur J Clin Nutr 2007;61:1400-6.

30. Bauer DC, Sklarin PM, Stone KL, Black DM, Nevitt MC, Ensrud KE, et al. Biochemical markers of bone turnover and prediction of hip bone loss in older women: the study of osteoporotic fractures. J Bone Miner Res 1999; 14:1404-10.

31. Dresner-Pollak R, Parker RA, Poku M, Thompson J, Seibel MJ, Greenspan SL. Biochemical markers of bone turnover reflect femoral bone loss in elderly women. Calcif Tissue Int 1996:59:328-33.

32. Garnero P, Sornay-Rendu E, Duboeuf F, Delmas PD. Markers of bone turnover predict postmenopausal forearm bone loss over 4 years: the OFELY study. J Bone Miner Res 1999;14:1614-21.

33. Iki M, Morita A, Ikeda Y, Sato Y, Akiba T, Matsumoto T, 
et al. Biochemical markers of bone turnover predict bone loss in perimenopausal women but not in postmenopausal women - the Japanese Population-based Osteoporosis (JPOS) Cohort Study. Osteoporos Int 2006;17: 1086-95.

34. Keen RW, Nguyen T, Sobnack R, Perry LA, Thompson PW, Spector TD. Can biochemical markers predict bone loss at the hip and spine?: a 4-year prospective study of 141 early postmenopausal women. Osteoporos Int 1996; 6:399-406.

35. Welch A, Bingham S, Camus J, Dalzell N, Reeve J, Day $\mathrm{N}$, et al. Calcaneum broadband ultrasound attenuation relates to vegetarian and omnivorous diets differently in men and women: an observation from the European Prospective Investigation into Cancer in Norfolk (EPICNorfolk) population study. Osteoporos Int 2005;16:5906.
36. Seibel MJ. Clinical use of markers of bone turnover in metastatic bone disease. Nat Clin Pract Oncol 2005;2: 504-17.

37. Kim GS, Kim CH, Park JY, Lee KU, Park CS. Effects of vitamin B12 on cell proliferation and cellular alkaline phosphatase activity in human bone marrow stromal osteoprogenitor cells and UMR106 osteoblastic cells. Metabolism 1996;45:1443-6.

38. Herrmann M, Schmidt J, Umanskaya N, Colaianni G, AlMarrawi F, Widmann T, et al. Stimulation of osteoclast activity by low B-vitamin concentrations. Bone 2007;41: 584-91.

39. Herrmann M, Tami A, Wildemann B, Wolny M, Wagner A, Schorr $\mathrm{H}$, et al. Hyperhomocysteinemia induces a tissue specific accumulation of homocysteine in bone by collagen binding and adversely affects bone. Bone 2009;44:467-75. 